\title{
Interpretation of quantitative crystallographic texture in copper electrodeposits on amorphous substrates
}

Pantleon, Karen; Jensen, Jens Arne Dahl; Somers, Marcel A. J.

Published in:

Journal of The Electrochemical Society

Link to article, DOI:

$10.1149 / 1.1628236$

Publication date:

2004

Document Version

Publisher's PDF, also known as Version of record

Link back to DTU Orbit

Citation (APA):

Pantleon, K., Jensen, J. A. D., \& Somers, M. A. J. (2004). Interpretation of quantitative crystallographic texture in copper electrodeposits on amorphous substrates. Journal of The Electrochemical Society, 151(1), C45-C51. https://doi.org/10.1149/1.1628236

\section{General rights}

Copyright and moral rights for the publications made accessible in the public portal are retained by the authors and/or other copyright owners and it is a condition of accessing publications that users recognise and abide by the legal requirements associated with these rights.

- Users may download and print one copy of any publication from the public portal for the purpose of private study or research.

- You may not further distribute the material or use it for any profit-making activity or commercial gain

- You may freely distribute the URL identifying the publication in the public portal 


\title{
Interpretation of Quantitative Crystallographic Texture in Copper Electrodeposits on Amorphous Substrates
}

\author{
Karen Pantleon, ${ }^{\mathrm{Z}}$ Jens A. D. Jensen, and Marcel A. J. Somers \\ The Technical University of Denmark, Department of Manufacturing Engineering and Management, \\ Lyngby, Denmark
}

\begin{abstract}
Crystallographic texture and morphology in $\mathrm{Cu}$ electrodeposits was studied in relation to the current density and the content of the organic levelling additive 3-mercapto-1-propanesulfonate. The substrate onto which $\mathrm{Cu}$ was electrodeposited consisted of amorphous Ni-P in order to allow substrate-unbiased texture development in the electrodeposit. Comprehensive X-ray diffraction studies of the crystallographic texture, including calculations of the three-dimensional orientation distribution function, were performed and accompanied by investigations of the deposit morphology by means of light optical microscopy. Electrodeposits with totally different microstructures having the same main crystallographic orientation of grains were observed. For interpretation of the results, not only the original growth behavior in direct dependence on the applied process parameters, but also process dependent self-annealing effects (recrystallization) of the as-deposited layers were considered.

(C) 2003 The Electrochemical Society. [DOI: 10.1149/1.1628236] All rights reserved.
\end{abstract}

Manuscript submitted February 4, 2003; revised manuscript received July 3, 2003. Available electronically December 9, 2003.

Tailoring the properties of electrodeposits requires a thorough understanding of the relation between the parameters of electrodeposition from aqueous solution, the internal structure of the deposit, and the associated physical and mechanical properties. Important process parameters concern both the condition of the electrochemical bath as well as the condition of the substrate. For the electrolyte: composition, temperature, current density at the cathode, and transport properties are of main importance; for the substrate (cathode): the crystallography and surface topography have a strong influence on the deposit. Several of these parameters cannot be changed independently, because synergetic effects need to be taken into account. For example, changing the electrolyte composition may require a modification of the current density for yielding an optimum effect; ${ }^{1}$ the efficiency of organic levelling and brightening additives in inhibiting growth in the thickness direction and promoting lateral growth can be improved by optimizing the current density. These several process parameters determine the nucleation and growth mode of the electrodeposit, as is reflected by the microstructure, i.e., grain size distribution and grain orientation distribution, in the deposit. Consequently, preferred crystal orientations of grains develop during electrodeposition, leading to characteristic growth textures.

For $\mathrm{Cu}$ layers (and some other face-centered cubic materials, too), characteristic growth textures can only be studied immediately after deposition, because the nonequilibrium state of the deposit changes even at room temperature. Two stages can be distinguished in this so-called self-annealing: recovery and recrystallization. ${ }^{\mathrm{a}, 2}$ During recovery, the rearrangement of point and line defects leads to the development of subgrains, which is not associated with dramatic changes in crystallographic texture. During recrystallization, a new microstructure (size and orientation distribution of the constituting grains) evolves by nucleation and grain boundary migration. Both self-annealing effects can be investigated with X-ray diffraction (XRD). While recovery affects the widths and positions of X-ray peak profiles, recrystallization additionally changes the crystallographic texture as reflected by the spatial distributions of peak profile intensities. Dramatic changes in preferred grain orientations were observed for $\mathrm{Cu}$ layers over a period of hours or weeks after electrochemical deposition. ${ }^{3-5}$ Consequently, the properties of $\mathrm{Cu}$ electrodeposits, applied e.g., as interconnect lines in microelectronics, change due to the course of self-annealing; a decrease of internal stress level $^{6}$ and microhardness ${ }^{7,8}$ as well as electrical resistivity $6,9,10$ have been reported.

A direct comparison of crystallographic texture of electrodepos-

\footnotetext{
${ }^{\text {z } E-m a i l: ~ p a n t l e o n @ i p l . d t u . d k ~}$
}

${ }^{\text {a }}$ In principle, a third step has to be mentioned: anomalous grain growth (or secondary recrystallization). its reported in the literature is difficult, if possible at all, because the interaction between very different electrolyte compositions and deposition parameters as well as the extent of self-annealing has to be considered. Additionally, the method pursued for (quantitative) texture determination, largely determines the significance of the result obtained. Often, the crystallographic textures reported as the result of XRD investigations are experimental pole figures only or even the intensity ratios for several diffraction peaks with respect to each other. This may lead to serious misinterpretation if the actual texture present in the deposit deviates from an ideal fiber texture. Furthermore, usually only the texture components corresponding to low index lattice planes as $\langle 111\rangle,\langle 100\rangle,\langle 110\rangle$ are given, while other components, which may be characteristic for recrystallization textures, are omitted. However, a thorough investigation of the crystallographic texture is essential for understanding the growth of the electrodeposit and for the prediction of texture related properties. Therefore, quantification of the crystallographic texture in terms of the orientation distribution function (ODF) ${ }^{11}$ is necessary. The ODF is not directly measurable by diffraction methods, but it can be calculated from experimental pole figures.

The present paper focuses on crystallographic texture and morphology in $\mathrm{Cu}$ electrodeposits in relation to the current density and the content of the organic levelling additive 3-mercapto-1propanesulfonate (MPSA). The applied electrolyte contains the same combination of additives as recently designed for superconformal filling of $\mathrm{Cu}$-interconnect structures in ultra-large-scale integrated features; ${ }^{9}$ but higher MPSA concentrations are applied here. The substrate onto which $\mathrm{Cu}$ was electrodeposited consisted of $\mathrm{X}$-ray amorphous Ni-P in order to minimize biasing the developing texture in the electrodeposit. Comprehensive XRD studies of the crystallographic texture, including calculations of the threedimensional (3-D) ODF, were performed and accompanied by investigations of the deposits morphology by means of light optical microscopy.

\section{Experimental}

Electrochemical deposition.-Copper layers were deposited from acidic electrolytes. The basis composition is $0.56 \mathrm{~mol} / \mathrm{L} \mathrm{CuSO}_{4} \times 5 \mathrm{H}_{2} \mathrm{O}, \quad 1.43 \mathrm{~mol} / \mathrm{L} \mathrm{H}_{2} \mathrm{SO}_{4}$, and 1.13 $\times 10^{-3} \mathrm{~mol} / \mathrm{L} \mathrm{Cl}^{-}$. In addition the electrolyte contains two organic additives: $8.82 \times 10^{-5} \mathrm{~mol} / \mathrm{L}$ polyethylene glycol (PEG, average molar mass of $3400 \mathrm{~g} / \mathrm{mol}$ ) and three levels of MPSA: 0.5 $\times 10^{-3} \mathrm{M}, 1.0 \times 10^{-3} \mathrm{M}$ and $1.5 \times 10^{-3} \mathrm{M}$. While the combination of $\mathrm{Cl}$ and PEG provides significant inhibition of the deposition reaction, MPSA leads to an acceleration of the deposition rate by displacing the more weakly bound inhibiting species on the deposit surface. ${ }^{9,12-14}$ For each electrolyte composition three current 


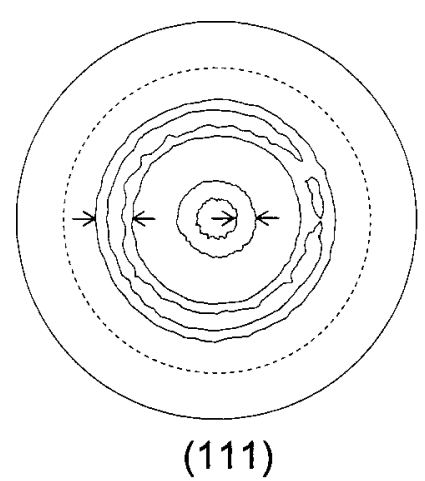

(111)

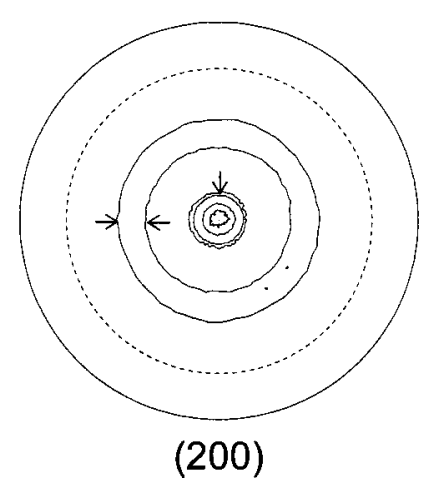

(200)

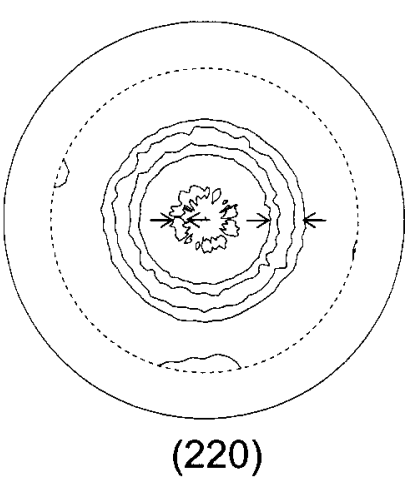

Figure 1. Pole figures (corrected for background and defocusing) for a $\mathrm{Cu}$ layer deposited on an amorphous Ni-P substrate from the electrolyte containing $1.0 \times 10^{-3} \mathrm{M}$ MPSA additive at a current density of $9 \mathrm{~A} / \mathrm{dm}^{2}$. The dashed circles indicate the tilt angle $\psi=75^{\circ}$ beyond which measurement is not possible. Arrows indicate the direction of increasing level starting from random. densities $\left(1,3\right.$, and $\left.9 \mathrm{~A} / \mathrm{dm}^{2}\right)$ were applied during deposition, giving nine possible combinations of current density and electrolyte composition.

The current density distribution was calculated using the electrochemical modeling software ElSyCa and optimized by applying a polypropylene mask in front of the cathode to obtain an equal thickness distribution of the electrodeposit over the entire substrate area. The calculation with ElSyCa was based on the following: For the anode, a linear relationship between current density and applied potential was assumed. For the cathode, a Butler-Volmer type dependency, $J=J_{0} \exp (\alpha n F / R T)-J_{0} \exp (-\beta n F / R T)(J$ is current flux, $\alpha$ is charge transfer coefficient, $b$ is $1-\alpha, n$ is number of electrons taking part in electrochemical reaction, $F$ is Faraday's constant, $R$ is the gas constant, and $T$ is temperature), was adopted. The distance between cathode and mask was set to $50 \mathrm{~mm}$. For more details about the current density simulation, see Ref. 15 .

The deposition time for each current density was taken as that corresponding to a uniform layer thickness of $15 \mu \mathrm{m}$. Deposition was carried out at room temperature with vigorous air agitation of the electrolyte.

The $\mathrm{Cu}$ layers were deposited onto amorphous Ni-P layers, obtained by electroless plating onto mild steel sheets. This allows the study of substrate-unbiased growth of the $\mathrm{Cu}$ deposits.

The samples were stored at room temperature for a period of about two months after deposition. It is anticipated that a stationary microstructure has evolved in the deposits as a consequence of selfannealing during storage. For selected deposition conditions, additional samples were investigated as-deposited for comparison purposes. The evolution of the crystallographic texture during storage will be the subject of future investigations.

XRD. - X-ray measurements were performed with a D8 Discover powder diffractometer (Bruker AXS). The X-ray tube is combined with a Göbel mirror resulting in a highly parallel incident beam with high intensity. The goniometer was equipped with a 1/4-circle Euler cradle, allowing accurate sample tilt and rotation as needed for texture analysis. X-ray measurements were carried out with $\mathrm{Cu} \mathrm{K \alpha}$ radiation. The corresponding information depth in $\mathrm{Cu}$ layers amounts to (maximally) $5 \mu \mathrm{m}$.

In order to study the crystallographic texture in $\mathrm{Cu}$ deposits with XRD, the intensities of (111), (200), and (220) diffracting lattice planes were recorded for various orientations of the diffraction vector with respect to the sample normal by changing the sample orientation over defined sample tilts $\left(0 \leqslant \psi \leqslant 75^{\circ}\right.$ at steps of $\left.5^{\circ}\right)$ and rotations $\left(0 \leqslant \varphi \leqslant 360^{\circ}\right.$ at steps of $\left.5^{\circ}\right)$. The measurement time per step of sample tilt and rotation was 6 s. All measured intensities were background corrected. A textureless, fine grained $\mathrm{Cu}$ reference sample was used for defocusing correction. Intensity distributions were summarized in pole figures and displayed as contour plots in stereographic projections.

For a thorough quantification of preferred crystal orientations, the 3-D ODF was calculated from the (111), (200), and (220) pole figures. For calculation of the ODF the series expansion, using harmonic functions ${ }^{11}$ with rank 34 and a positivity refinement was ap- plied by recalculating two supplementary pole figures (331) and (311) in order to avoid the determination of virtual texture components, so-called ghosts. While measured pole figures are always incomplete because the measurement in reflection mode is restricted to tilt angles of $75^{\circ}$, complete pole figures were obtained from the ODF by calculating the missing area between $75^{\circ}$ and $90^{\circ}$. Additionally, inverse pole figures were obtained from the ODF.

Inverse pole figures in three sample directions (normal direction and two mutually perpendicular directions within the plane of the deposit) indicate the frequency with which different crystal directions occur in a specific sample direction. For ideal fiber textures, the inverse pole figure along the fiber axis is identical to the complete $3-\mathrm{D} \mathrm{ODF}^{11}$ and inverse pole figures in directions within the plane of the deposit represent random rotations around the fiber axis. Maxima in the inverse pole figures in normal direction to the substrate were used to determine the fiber axis $\langle\mathrm{uvw}\rangle$ and the corresponding orientation densities as a measure of the strength of the texture.

Microscopy.-To study the evolution of the morphology from the interface with the Ni-P substrate to the surface of the electrodeposit, cross sections of the samples were prepared and investigated using a Neophot 30 (Carl Zeiss, Jena) light optical microscope (LOM). The samples were etched in a freshly prepared 1:1:1 mixture of $\mathrm{NH}_{4} \mathrm{OH}, \mathrm{H}_{2} \mathrm{O}_{2}$, and distilled water. Bright field images were taken at a resolution of about $200 \mathrm{~nm}$, using a planapochromate oil immersion and monochromatic (green) light objective.

\section{Results}

Crystallographic texture.-Measured pole figures of $\mathrm{Cu}$ deposits on amorphous Ni-P substrates are completely rotationally symmetric around normal direction. No inclinations of the fiber axis were observed, indicating that all $\mathrm{Cu}$ deposits on amorphous Ni-P substrates developed ideal fiber textures, in contrast to the deposition of $\mathrm{Cu}$ layers on polycrystalline $\mathrm{Cu}$ substrates. ${ }^{16}$ By means of example, the pole figures for a $\mathrm{Cu}$ layer deposited with $1.0 \times 10^{-3} \mathrm{M}$ MPSAadditive content and current density of $9 \mathrm{~A} / \mathrm{dm}^{2}$ are shown in Fig. 1.

The development of ideal fiber textures, e.g., fiber axis exactly parallel to the surface normal is expected for electrodeposits on amorphous substrates. In contrast, restricted fiber textures, where the fiber axes are inclined to the surface, as generally observed for films grown by physical vapor deposition, ${ }^{17}$ can develop during electrodeposition only as a consequence of epitaxy between deposit and polycrystalline substrate with orthorhombic texture or when the electric field lines are inclined to the surface normal, e.g., at edges and corners. In case of epitaxy, restricted fiber textures represent a transition zone between orthorhombic textures, due to substrate influence, and the intrinsic ideal fiber textures developing for a thick deposit, as found for $\mathrm{Cu}$ electrodeposits on rolled $\mathrm{Cu}$ substrates with orthorhombic texture. ${ }^{18}$

Inverse pole figures along the surface normal, i.e., the direction of film growth, were used for quantification of fiber textures. For the electrodeposits, it was observed that inverse pole figures within the 


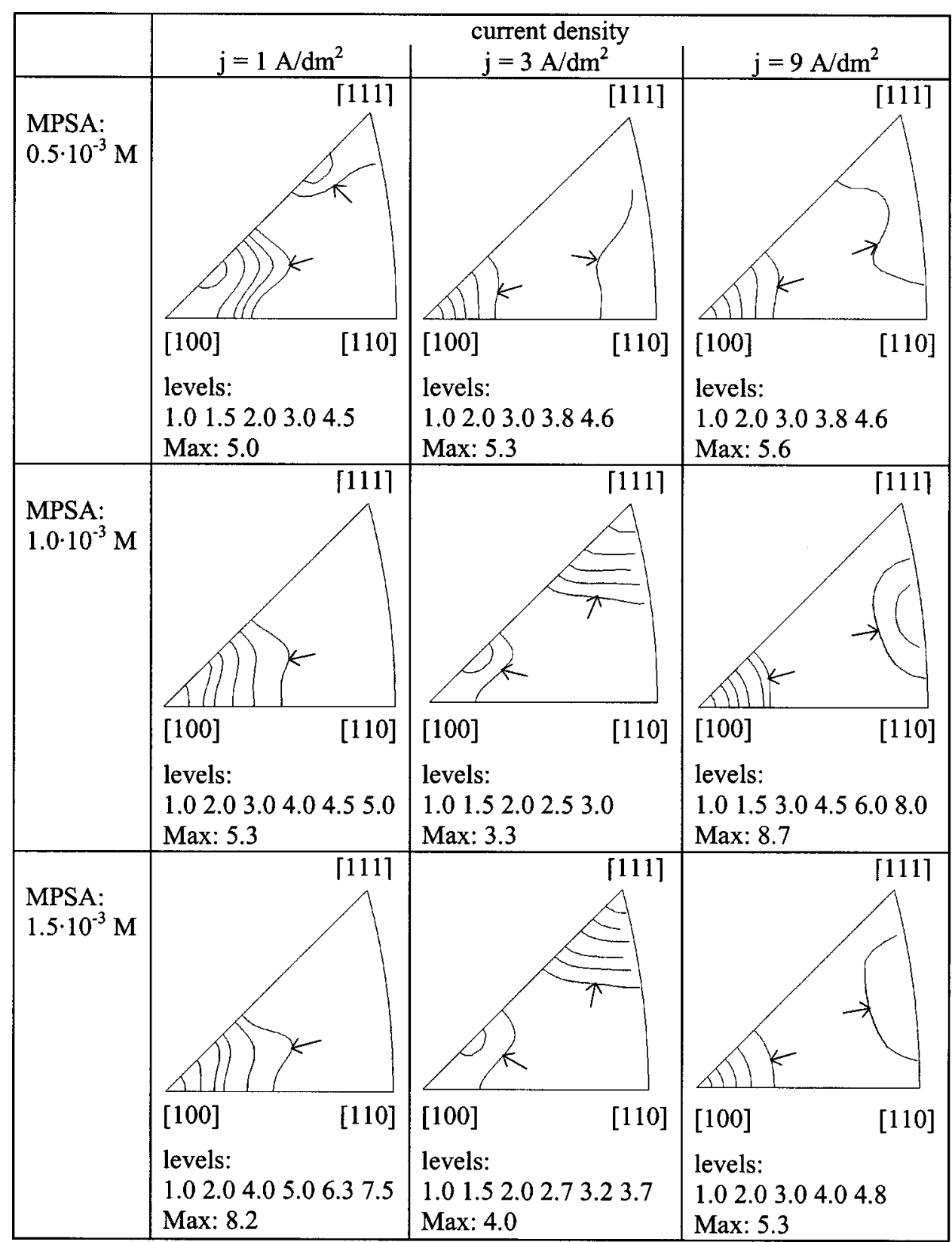

Figure 2. Inverse pole figures in normal direction for $\mathrm{Cu}$ layers deposited on the amorphous Ni-P substrates for three MPSA additive contents at three current densities. Measurements were performed with $\mathrm{Cu} \mathrm{K} \mathrm{K}_{\alpha}$ radiation. Arrows indicate the direction of increasing level starting from random. plane of the layer were almost identical to each other and their orientation densities were close to one, implying random orientation in these directions, as expected for ideal fiber textures. Inverse pole figures in the normal direction, as obtained from ODF calculations, are shown for all combinations of deposition parameters in Fig. 2.

The positions of the maxima of the orientation density in the inverse pole figures in normal direction are summarized for all samples in Fig. 3 and assigned to the corresponding directions, e.g., the fiber axes.

Table I summarizes the texture components, i.e., the maxima in the inverse pole figures, and the corresponding orientation densities of $\mathrm{Cu}$-electrodeposits on amorphous Ni-P substrates as obtained from inverse pole figures along the normal direction (cf. also Fig. 2 and Fig. 3).

For $\mathrm{Cu}$ layers on amorphous Ni-P substrates, both the current density and the additive content significantly influenced the orientation densities. At a low current density of $1 \mathrm{~A} / \mathrm{dm}^{2}$, a $\langle 100\rangle$ fiber texture developed with increasing MPSA-additive content to a rather strong single texture component. Only at low additive content this component competed with a $\langle 611\rangle$ texture, which is relatively close to $\langle 100\rangle$ (but not related to an inclination of the $\langle 100\rangle$ fiber axis, as obvious from the ideal rotational symmetry of the pole figures). A medium current density of $3 \mathrm{~A} / \mathrm{dm}^{2}$ resulted at low additive concentration in a strong $\langle 100\rangle$ fiber texture. However, the $\langle 100\rangle$ decreased dramatically with increasing additive content and concurrently a $\langle 111\rangle$ fiber texture developed. As a result, at high additive content of $1.5 \times 10^{-3} \mathrm{M}$, the main texture component was $\langle 111\rangle$ while the $\langle 100\rangle$ had vanished. The development of $\langle 111\rangle$ was accompanied by a minor $\langle 511\rangle$ component. At a current density of $9 \mathrm{~A} / \mathrm{dm}^{2}$, the dominant component was again $\langle 100\rangle$ and simultaneously, a $\langle 221\rangle$ texture developed with increasing concentration of MPSA additive.

The results show that a medium current density $\left(3 \mathrm{~A} / \mathrm{dm}^{2}\right)$ has a special importance, because it changes not only the texture strength, but also a new type of texture develops with increasing additive concentration. Furthermore, it can be concluded that additional weak texture components are formed at low MPSA content, irrespective of the current density. These components appear to be suppressed at higher MPSA contents. 


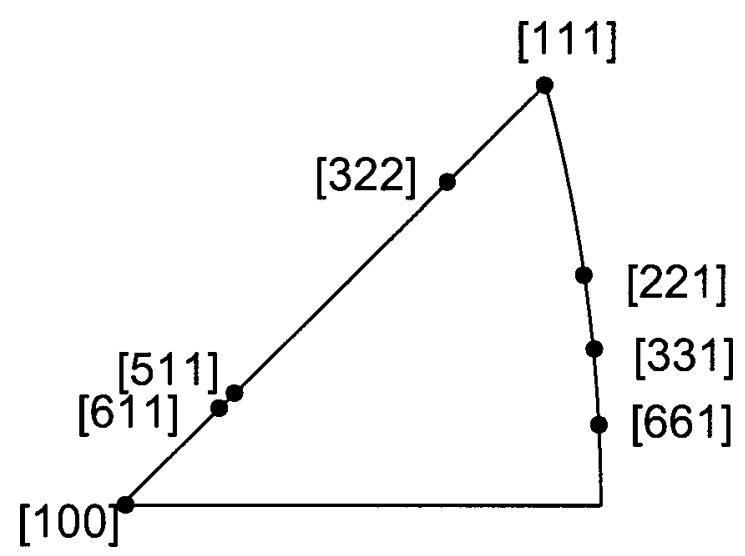

Figure 3. Schematic inverse pole figure, summarizing observed maxima of the orientation density and the corresponding directions normal to the sample surface.

The results show that the texture of electrodeposits can only be described thoroughly by means of ODF calculations from measured XRD pole figures. That is essential in order to quantify not only main texture components but also minor ones, including $\langle 511\rangle$ and $\langle 221\rangle$ twin orientations. It should be emphasized that $\langle 221\rangle$ and $\langle 511\rangle$ texture components are not artifacts from ODF calculations, they are already visible in the experimental pole figures. For example, in Fig. 1, the $\langle 100\rangle$ orientation is clearly seen in the center of the (200) pole figure as well as at $54.7^{\circ}$ in (111) and $45.0^{\circ}$ in (220). From additional maxima of the pole density, at $48.2^{\circ}$ in the (200) pole figure [and at $15.8^{\circ}$ in (111) and at $19.5^{\circ}$ in (220)], it can be concluded that there must be a fraction of grains with (221) lattice planes parallel to the surface forming a $\langle 221\rangle$ fibre texture. In a similar way, the existence of the $\langle 511\rangle$ (and all other higher indexed components) can be proved by inspection of the corresponding experimental pole figures of low-indexed lattice planes.

Morphology.-The grain size distribution in the deposited layers was strongly influenced by the applied current density, while increasing the MPSA concentration in the electrolyte had a less pronounced effect. ${ }^{15}$ By means of example, the microstructures in $\mathrm{Cu}$ layers deposited at the three current densities for medium MPSAadditive content of $1.0 \times 10^{-3} \mathrm{M}$ are discussed (Fig. 4).

The deposited layer thickness decreased slightly with current density, even though the total transferred charge to the sample was equal for all samples and would correspond to a layer thickness of $15 \mu \mathrm{m}$ for $100 \%$ efficiency.

Close to the interface, very small grains are observed for the low and medium current density $\mathrm{Cu}$ electrodeposits; grain sizes were less than $1 \mu \mathrm{m}$ (Fig. $4 \mathrm{a}$ and b). The microstructure changed significantly within the first 3-4 $\mu \mathrm{m}$ away from the substrate. At low

Table I. Texture components and orientation densities of $\mathrm{Cu}$ electrodeposits on amorphous Ni-P substrates. Only texture components with orientation densities larger than 1 are given; a value of 1 represents an abundance conforming to random orientation.

Current density

\begin{tabular}{lccc}
\hline $\begin{array}{l}\text { MPSA } \\
\text { additive content }\end{array}$ & $\begin{array}{c}1 \mathrm{~A} / \mathrm{dm}^{2} \\
(\text { low })\end{array}$ & $\begin{array}{c}3 \mathrm{~A} / \mathrm{dm}^{2} \\
(\text { medium })\end{array}$ & $\begin{array}{c}9 \mathrm{~A} / \mathrm{dm}^{2} \\
(\mathrm{high})\end{array}$ \\
$\begin{array}{l}\left\langle 6.5 \times 10^{-3} \mathrm{M}\right. \\
(\text { low })\end{array}$ & $\langle 611\rangle 5.0$ & $\langle 100\rangle 5.3$ & $\langle 100\rangle 5.6$ \\
& $\langle 322\rangle 1.7$ & $\langle 322\rangle 1.1$ & $\langle 322\rangle 1.2$ \\
$1.0 \times 10^{-3} \mathrm{M}$ & $\langle 100\rangle 5.3$ & $\langle 111\rangle 3.3$ & $\langle 100\rangle 8.7$ \\
$($ medium $)$ & & $\langle 511\rangle 1.7$ & $\langle 221\rangle 1.8$ \\
& $\langle 100\rangle 8.2$ & $\langle 111\rangle 4.0$ & $\langle 100\rangle 5.3$ \\
$1.5 \times 10^{-3} \mathrm{M}$ & & $\langle 511\rangle 1.6$ & $\langle 221\rangle 1.5$ \\
$($ high $)$ & & &
\end{tabular}

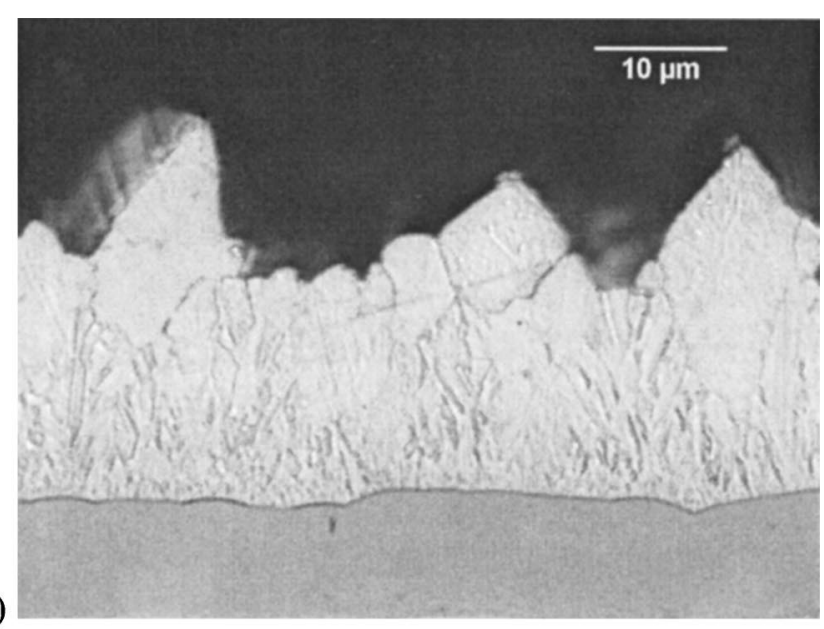

a)

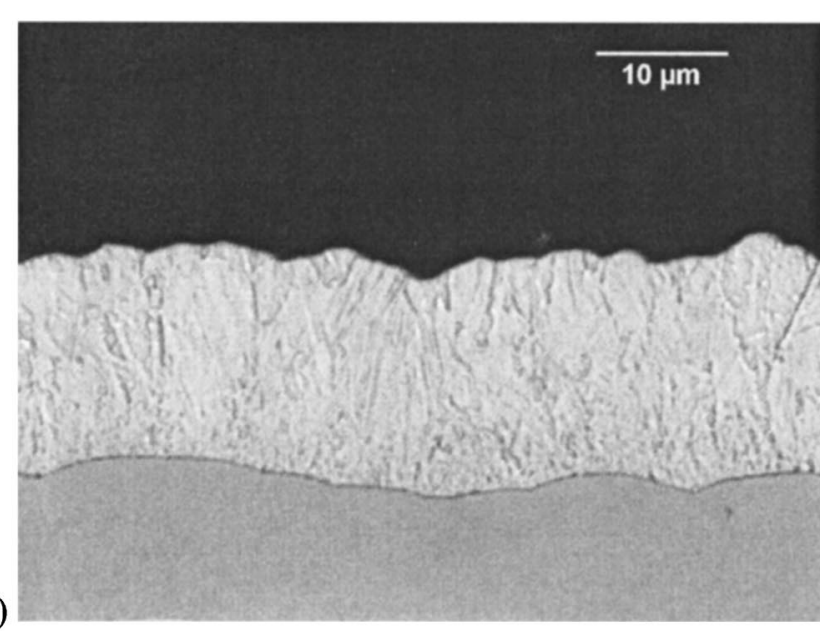

b)

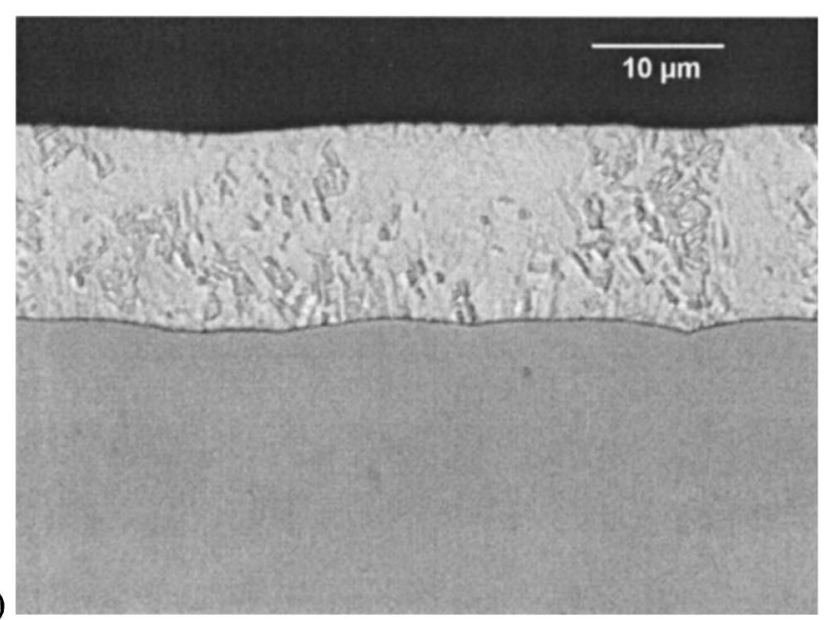

Figure 4. Microstructure observed with LOM of $\mathrm{Cu}$ layers deposited with MPSA-additive content of $1.0 \times 10^{-3} \mathrm{M}$ and current density of (a) $1 \mathrm{~A} / \mathrm{dm}^{2}$, (b) $3 \mathrm{~A} / \mathrm{dm}^{2}$, and (c) $9 \mathrm{~A} / \mathrm{dm}^{2}$.

current density $\left(1 \mathrm{~A} / \mathrm{dm}^{2}\right)$, some of the small grains became elongated in the growth direction and coarsened to grain diameters exceeding $10 \mu \mathrm{m}$ at the surface. This preferential outward growth of some of the grain orientations resulted in rough surfaces. Concurrent with this growth selection, the formation of twins was observed (see Fig. 4a). However, twins were not observed in the crystals protruding from the surface. At medium current density $\left(3 \mathrm{~A} / \mathrm{dm}^{2}\right)$ grain coarsening occurred as well, but grain sizes amounted to only a few 
Table II. Primary twin directions calculated for all observed fiber texture components applying a rotation over $60^{\circ}$ about [111].

Fiber axis

$\langle 100\rangle$

$\langle 111\rangle$

$\langle 611\rangle$

$\langle 511\rangle$

$\langle 322\rangle$

$\langle 221\rangle$

$\langle 661\rangle$

$\langle 331\rangle$

micrometers, even at the surface. Additionally, a relatively high density of twins was observed in Fig. 4b. For the high current density deposit, the $\mathrm{Cu}$ grains adjacent to the substrate appeared coarser than for the low and medium current density deposits (Fig. 4c). Moreover, the microstructure was uniform and relatively fine grained throughout the deposit; no single grains could have been resolved. This indicates that the outgrowth of preferred crystallographic orientations is effectively suppressed, thus leading to smooth surfaces without protuberant crystallites. Due to the resolution limit of light optical microscopy, it was not possible to determine the twin density of these small grains; only a few larger twins were resolved (see Fig. 4c).

\section{Discussion}

Comparison of crystallographic texture and morphology.-The minor texture components $\langle 511\rangle$ and $\langle 221\rangle$ at medium and high current density (Table I) can be explained from twinning of $\langle 111\rangle$ and $\langle 100\rangle$ oriented grains, respectively. Twinning occurs readily because of the relatively low stacking fault energy of copper. In face centered cubic crystals, twinning of a crystallographic direction is represented by a rotation over $60^{\circ}$ about [111]. This implies for the

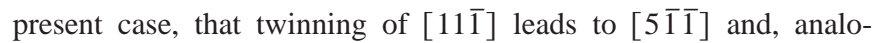
gously, twinning of [010] yields [221] . Consequently, for fiber textures, twinning in grains belonging to the fiber texture introduces a new fiber component. Accordingly, the $\langle 511\rangle$ fiber texture evolves from twinning of $\langle 111\rangle$ oriented grains and the $\langle 221\rangle$ fiber texture develops from twinning of $\langle 100\rangle$ oriented grains. In Table II, primary twin directions are calculated for all observed fiber axes. It can be concluded that measured fiber textures $\langle 611\rangle,\langle 322\rangle,\langle 661\rangle$, and $\langle 331\rangle$ do not result from twinning, because the required original (parent) direction is unlikely to occur for electrodeposits, e.g., $\langle 20.16 .1\rangle$ for the observed $\langle 661\rangle$, and indeed not detected experimentally.

The occurrence of twinning is consistent with micrographs in Fig. $4 \mathrm{~b}$ and $\mathrm{c}$ for medium and high current density. Also, twinning was observed in the continuous part of the layer deposited at low current density, see Fig. 4a; however no twinned grains belonging to the fiber texture were identified during texture analysis. The absence of crystallographic evidence for twins in the low current density deposits is attributed to the presence of the relatively large, protruding crystals (Fig. 4a), which are virtually free of twins and dominate the $\langle 100\rangle$ component.

In Ref. 3,19, multiple twinning has been reported as well. However, the present study did not provide evidence for the occurrence of multiple twinning in the electrodeposits.

The microstructures in Fig. $4 \mathrm{a}$ and $\mathrm{b}$ clearly indicate that preferred grain orientations at low and medium current density changed from the interface with Ni-P to the surface. The microstructure of the high current density deposit, Fig. 4c, did not allow any conclusions about the texture. Indeed, in Ref. 20, it was reported that $\mathrm{Cu}$ layers on amorphous Ni-P grew from random grain orientation in 3 $\mu \mathrm{m}$ thick electrodeposits. This strongly indicates that nucleation of $\mathrm{Cu}$ onto Ni-P is unbiased by the substrate, in the sense that the orientation distribution of $\mathrm{Cu}$ crystals is random. On continued electrodeposition, preferred grain orientations evolve according to growth selection processes in the direction of the electric field developing a fiber axis in the growth direction, with fiber axis type typical for the applied conditions of electrodeposition. The investigation of texture gradients was not the objective of the present study. However, texture analysis with a shallower penetrating $\mathrm{Cr} \mathrm{K} \alpha$ radiation (information depth amounts to about $2 \mu \mathrm{m}$ ) was performed as a supplement to the results obtained with $\mathrm{Cu} \mathrm{K} \alpha$ radiation. Texture measurements with $\mathrm{Cr} \mathrm{K} \alpha$ radiation gave almost identical (quantitative) results for the main texture components as obtained from investigations with $\mathrm{Cu} K \alpha$ radiation. However, as minor texture components only the well-defined twin orientations $\langle 511\rangle$ and $\langle 221\rangle$ were identified. The additional minor components as observed with $\mathrm{Cu} \mathrm{K} \alpha$ for the low additive content deposits (see Table I) remained unobserved with $\mathrm{Cr} \mathrm{K} \alpha$.

Origin of texture in $\mathrm{Cu}$ electrodeposits: growth vs. recrystallization texture.-The results in Table I and Fig. 2 show that the $\langle 100\rangle$ fiber texture occurred as the main texture component with high orientation density, both at low $\left(1 \mathrm{~A} / \mathrm{dm}^{2}\right)$ and high $(9$ $\mathrm{A} / \mathrm{dm}^{2}$ ) current density, regardless of the MPSA-additive level. In contrast, the microstructure and the surface topography were totally different for these current densities: very rough surfaces resulting from large grains elongated in the growth direction and very finegrained microstructures with smooth surfaces respectively (compare Fig. $4 \mathrm{a}$ and c). For interpretation of the obtained results, also minor texture components have to be considered. In case of low current density $\left(1 \mathrm{~A} / \mathrm{dm}^{2}\right)$, a strong single fiber texture of $\langle 100\rangle$ type was measured without any additional components. However, at high current density $\left(9 \mathrm{~A} / \mathrm{dm}^{2}\right)$, in addition to $\langle 100\rangle$ a weaker $\langle 221\rangle$ was observed as a twinning orientation.

The texture developing during layer growth (growth texture) is mainly controlled by the surface energy, the strain energy stored in the layer and the mobility of adsorbed atoms on the surface. For surface energy minimization reasons the development of grains bounded by close-packed lattice planes at the substrate surface is favored. Consequently, for $\mathrm{Cu}$ the lowest surface energy is expected for a flat surface and a $\langle 111\rangle$ fiber texture. ${ }^{21}$ The presence of additives at the interface between the electrodeposit and the electrolyte modifies the surface energy and, accordingly, may cause a change of texture. Alternatively, the incorporation of additives in the growing deposit affects the distribution of lattice defects in the deposit, leading to modifications of the strain distribution. Then, also other fiber axes can become favourable. For example, a $\langle 100\rangle$ fiber axis develops for conditions that favor strain energy minimization..$^{22,23}$

Notwithstanding the type of growth texture, the simultaneous occurrence of both $\langle 111\rangle$ and $\langle 100\rangle$ and their corresponding twins, e.g., $\langle 511\rangle$ or $\langle 221\rangle$ appears unlikely as the result of growth selection. Rather, the occurrence of the fiber axes $\langle 511\rangle$ and $\langle 221\rangle$ in twin relation are regarded as the outcome of recrystallization. Consequently, the double fiber textures with simultaneous presence of the parent and twin orientation $(\langle 111\rangle+\langle 511\rangle$ and $\langle 100\rangle+\langle 221\rangle)$ are considered as recrystallization textures. In order to check this interpretation, electrodeposition under identical conditions was repeated in a few selected cases and texture analysis of these equivalent samples was performed immediately after deposition. ${ }^{\mathrm{b}}$ The results of as-deposited samples with $1.5 \times 10^{-3}$ M MPSA are shown in Fig. 5. No texture components related to twins were found immediately after deposition; hence, observed twin orientations after sample storage can be considered as recrystallization twins developing with time.

From the discussion above it can be concluded that the strong $\langle 100\rangle$ fiber textures observed in low and high current density deposits (Table I) had different origins. At low current density, $\langle 100\rangle$ is considered the intrinsic growth texture of $\mathrm{Cu}$ layers, typical for the parameters applied during electrodeposition. This interpretation is in agreement with studies on an as-deposited sample (cf. Fig. 5a)

\footnotetext{
${ }^{b}$ It should be noted that it was not the objective of the present paper to study time-dependent changes of the texture. A limited number of as-deposited samples were investigated only to support the interpretation of the results.
} 


\begin{tabular}{|c|c|c|c|}
\hline & $\mathrm{j}=1 \mathrm{~A} / \mathrm{dm}^{2}$ & $\begin{array}{c}\text { current density } \\
\mathrm{j}=3 \mathrm{~A} / \mathrm{dm}^{2}\end{array}$ & $\mathrm{j}=9 \mathrm{~A} / \mathrm{dm}^{2}$ \\
\hline \multirow[t]{5}{*}{$\begin{array}{l}\text { MPSA: } \\
1.5 \cdot 10^{-3} \mathrm{M}\end{array}$} & a) & b) $\quad[111]$ & {$[111]$} \\
\hline & {$[100]$} & {$[110]$} & [110] \\
\hline & $\begin{array}{l}\text { levels: } \\
1.01 .52 .03 .04 .5 \\
\text { Max: } 4.9\end{array}$ & $\begin{array}{l}\text { levels: } \\
1.01 .251 .51 .752 .0 \\
\text { Max: } 2.2\end{array}$ & $\begin{array}{l}\text { levels: } \\
1.01 .52 .02 .53 .0 \\
\text { Max: } 3.4\end{array}$ \\
\hline & \multicolumn{3}{|c|}{ texture components, as-deposited } \\
\hline & $<100>4.9$ & $\begin{array}{l}<100>2.2 \\
<110>1.6\end{array}$ & $<100>3.4$ \\
\hline
\end{tabular}

Figure 5. Inverse pole figures in normal direction for $\mathrm{Cu}$ layers as-deposited. No maxima were detected for the twin orientations $\langle 221\rangle$ and $\langle 511\rangle$. Arrows indicate the direction of increasing level starting from random. showing indeed a $\langle 100\rangle$ growth texture. Texture analysis of samples after storage did not provide evidence for the presence of a twinning direction, so no recrystallization had occurred. This interpretation is further supported by the observed microstructure: the protuberances at the surface were single crystallites. Such features are not expected for a recrystallized microstructure, because recrystallization would lead to several grains within the protuberances. However, in the continuous part of the film deposited with low current density, where twins are visible, (partial) recrystallization may have occurred, but the contribution of this part of the layer to diffracted intensity in the texture measurements is limited.

In contrast, at high current density, self-annealing is likely to be completed: a $\langle 100\rangle$ texture is accompanied by a weaker $\langle 221\rangle$ texture component, resulting from twinning during recrystallization. From the as-deposited $\langle 100\rangle$ growth texture (cf. Fig. 5c) a recrystallization texture $\langle 100\rangle+\langle 221\rangle$ has developed. The uniform grain size throughout the layer (Fig. 4c) further supports the interpretation of completed recrystallization.

For medium current density, not only twin orientations as minor texture components indicate the onset of recrystallization, even a change of the main texture component was observed comparing the as-deposited sample at $1.5 \times 10^{-3} \mathrm{M}$ MPSA (growth texture: $\langle 100\rangle+\langle 110\rangle$, Fig. $5 b$ ) with the corresponding sample after storage (recrystallization texture: $\langle 111\rangle+\langle 511\rangle$ ).

Applying the outcome of the above discussion on the results collected in Table I it is concluded that recrystallization textures developed at medium and high current density $\left(3 \mathrm{~A} / \mathrm{dm}^{2}, 9 \mathrm{~A} / \mathrm{dm}^{2}\right)$ for medium and high MPSA-additive contents $\left(\geqslant 1.0 \times 10^{-3} \mathrm{M}\right)$. For all other deposition conditions the intrinsic growth textures remained unaffected, as shown exemplary for $j=1 \mathrm{~A} / \mathrm{dm}^{2}$ with $1.5 \times 10^{-3} \mathrm{M}$ MPSA comparing the texture components in an asdeposited sample with the sample after storage. Additives in the electrolyte enhanced the defect density of the deposit, ${ }^{24}$ which can be regarded as the driving force for recrystallization. According to this, fine-grained as-deposited microstructures, as generally obtained for high MPSA contents, promote recrystallization, while large initial grain sizes, as observed for deposits with low additive content, provided fewer nucleation sites for recrystallization. In this case, recrystallization took much longer to accomplish or occurs only at higher temperatures. Apart from the MPSA level also the current density played an important role, because additives were more effective (in terms of transport toward the surface and/or subsequent adsorption at the cathode surface) at higher current densities. Only after the MPSA-additive content exceeded a certain level $(\geqslant 1.0$ $\left.\times 10^{-3} \mathrm{M}\right)$, recrystallization happened at medium and high current density. For low current densities, where generally only low defect densities are generated, even the high MPSA content has not led to observable recrystallization during storage for about two months. Accordingly, the crystallographic orientation of the grains still reflects the intrinsic growth texture.

\section{Conclusions}

$\mathrm{Cu}$ layers electrodeposited onto amorphous Ni-P substrates from MPSA modified acidic copper electrolytes were investigated. The following conclusions can be drawn:

(i) Ideal fiber textures are formed due to the substrate unbiased growth.

(ii) Type and sharpness of texture depend both on the applied cathode current density and the concentration of MPSA additive in the electrolyte.

(iii) Microstructure and surface topography of the $\mathrm{Cu}$ layers are mainly influenced by the current density.

(iv) Electrodeposits with totally different microstructures can have the same main crystallographic orientation of the grains, and

(v) In addition to the original growth behavior determined by the applied process parameters, also process dependent self-annealing effects (recrystallization) in the as-deposited layers need to be considered for a full understanding of the microstructure in $\mathrm{Cu}$ electrodeposits.

In general, it can be stated that the properties of electrodeposits cannot be directly derived from their crystallographic texture only (cf. conclusion $i v$ ).

\section{Acknowledgment}

XRD studies presented here are part of a project financed by the Danish Technical Research Council (STVF). The authors gratefully acknowledge the financial support.

The Technical University of Denmark assisted in meeting the publication costs of this article.

\section{References}

1. R. Winand, Electrochim. Acta, 39, 1091 (1994).

2. F. J. Humphreys and M. Hatherly, Recrystallization and Related Annealing Phenomena, Pergamon Press, Elmsford, NY (1996).

3. C. Lingk, M. E. Gross, and W. L. Brown, J. Appl. Phys., 87, 2232 (2000)

4. Q.-T. Jiang, R. Mikkola, R. Ortega, and V. Blaschke, Mater. Res. Soc. Symp. Proc., 564, 393 (1999).

5. I. Handreg, P. Klimanek, G. Lange, and E. Weiland, in Proceedings of the 4th European Conference on Advanced Materials and Processes, Vol. 2, Associazione Italiana di Metallurgia, p. 471 (1995). 
6. S. H. Brongersma, E. Richard, I. Vervoort, H. Bender, W. Vandervorst, S. Lagrange, G. Beyer, and K. Maex, J. Appl. Phys., 86, 3642 (1999).

7. I. V. Tomov, D. S. Stoychev, and I. B. Vitanova, J. Appl. Electrochem., 15, 887 (1985).

8. S. Surnev and I. Tomov, J. Appl. Electrochem., 19, 752 (1989).

9. T. P. Moffat, J. E. Bonevich, W. H. Huber, A. Stanichevsky, D. R. Kelly, G. R. Stafford, and D. Josell, J. Electrochem. Soc., 147, 4524 (2000).

10. Q.-T. Jiang, R. Mikkola, B. Carpenter, and M. E. Thomas, Mater. Res. Soc. Symp. Proc., 1999, 177.

11. H.-J. Bunge, Texture Analysis in Materials Science: Mathematical Methods, Cuvillier Verlag, Göttingen (1993)

12. M. Hayase, M. Taketani, K. Aizawa, T. Hatsuzawa, and K. Hayabusa, Electrochem Solid-State Lett., 5, C98 (2002).

13. D. Josell, D. Wheeler, and T. P. Moffat, Electrochem. Solid-State Lett., 5, C49 (2002).

14. K. R. Hebert, J. Electrochem. Soc., 148, C726 (2001).
15. J. A. D. Jensen, Ph.D. Thesis, Dissertation no. 784, University of Linköping, Sweden, (2002).

16. K. Pantleon, J. D. Jensen, and M. A. J. Somers, Mater. Sci. Forum, 443-444, 205 (2004).

17. K. Fischer, Freiberger Forschungshefte B288, ISBN: 3-86012-055-7 (1998).

18. I. Handreg, Freiberger Forschungshefte B285, ISBN: 3-86012-050-6 (1998)

19. C. Schmidt, Galvanotechnik, 82, 3800 (1991).

20. A. A. Rasmussen, J. A. D. Jensen, A. Horsewell, M. A. J. Somers, Electrochim. Acta, 47, 67 (2001)

21. D. Udler and D. N. Seidmann, Phys. Rev. B, 54, R11133 (1996)

22. H. Lee, S. D. Lopatin, and S. S. Wong, in Proceedings of the Interconnect Technology Conference, IEEE, p. 114 (2000)

23. D. N. Lee, Mater. Sci. Forum, 408-412, 75 (2002).

24. M. E. Gross, R. Drese, D. Golovin, W. L. Brown, C. Lingk, S. Merchant, and M. Oh, Mater. Res. Soc. Symp. Proc., 2000, 85. 